OPEN ACCESS

Edited by:

Pinyi Lu,

Frederick National Laboratory for Cancer Research National Institutes of Health $(\mathrm{NIH})$

United States

Reviewed by:

Maria Soledad Ramirez, California State University, Fullerton,

United States

Xia $W u$,

University of Washington

United States

*Correspondence: Jing Zhang

zhangj_fudan@aliyun.com

${ }^{t}$ These authors have contributed equally to this work

Specialty section:

This article was submitted to Computational Genomics, a section of the journal Frontiers in Genetics

Received: 22 May 2020 Accepted: 14 April 2021 Published: 07 May 2021

Citation:

Li X, Liu X, Horvatovich P, Hu Y and Zhang J (2021) Proteomics

Landscape of Host-Pathogen

Interaction in Acinetobacter baumannii Infected Mouse Lung.

Front. Genet. 12:563516. doi: 10.3389/fgene.2021.563516

\section{Proteomics Landscape of Host-Pathogen Interaction in Acinetobacter baumannii Infected Mouse Lung}

\author{
Xin $\mathrm{Li}^{1,2,3+}$, Xiaofen Liu ${ }^{1,2,3 t}$, Peter Horvatovich4, Yingwei Hu${ }^{5}$ and Jing Zhang ${ }^{1,2,3 *}$ \\ ${ }^{1}$ Institute of Antibiotics, Huashan Hospital, Fudan University, Shanghai, China, ${ }^{2}$ Key Laboratory of Clinical Pharmacology \\ of Antibiotics, National Health and Family Planning Commission, Shanghai, China, ${ }^{3}$ National Clinical Research Center \\ for Aging and Medicine, Huashan Hospital, Fudan University, Shanghai, China, ${ }^{4}$ Department of Analytical Biochemistry, \\ Groningen Research Institute of Pharmacy, University of Groningen, Groningen, Netherlands, ${ }^{5}$ Department of Pathology, \\ Johns Hopkins University, Baltimore, MD, United States
}

Acinetobacter baumannii is an important pathogen of nosocomial infection worldwide, which can primarily cause pneumonia, bloodstream infection, and urinary tract infection. The increasing drug resistance rate of $A$. baumannii and the slow development of new antibacterial drugs brought great challenges for clinical treatment. Host immunity is crucial to the defense of $A$. baumannii infection, and understanding the mechanisms of immune response can facilitate the development of new therapeutic strategies. To characterize the system-level changes of host proteome in immune response, we used tandem mass tag (TMT) labeling quantitative proteomics to compare the proteome changes of lungs from $A$. baumannii infected mice with control mice $6 \mathrm{~h}$ after infection. A total of 6,218 proteins were identified in which 6,172 could be quantified. With threshold $p<0.05$ and relative expression fold change $>1.2$ or $<0.83$, we found 120 differentially expressed proteins. Bioinformatics analysis showed that differentially expressed proteins after infection were associated with receptor recognition, NADPH oxidase (NOX) activation and antimicrobial peptides. These differentially expressed proteins were involved in the pathways including leukocyte transendothelial migration, phagocyte, neutrophil degranulation, and antimicrobial peptides. In conclusion, our study showed proteome changes in mouse lung tissue due to $A$. baumannii infection and suggested the important roles of NOX, neutrophils, and antimicrobial peptides in host response. Our results provide a potential list of protein candidates for the further study of host-bacteria interaction in A. baumannii infection. Data are available via ProteomeXchange with identifier PXD020640.

Keywords: Acinetobacter baumannii, lung infection, host response, NADPH oxidase, TMT-labeling proteomics

\section{INTRODUCTION}

Acinetobacter baumannii is a hazardous Gram-negative opportunistic pathogen of nosocomial infection that represent serious health risk worldwide. The most serious consequence of lung infection by $A$. baumannii is nosocomial pneumonia, which has high mortality rate. In recent years, the resistance of $A$. baumannii to antibiotics is prominently increasing 
(Hu et al., 2019), and the resistance rate against imipenem and meropenem in China were 73.6 and $75.1 \%$ in 2019, respectively ${ }^{1}$. Although colistin is used as a last-line antibiotic against multiresistant A. baumannii, the nephrotoxicity of this compound greatly limited its clinical use (Li et al., 2006). The prevalence of resistant A. baumannii and the slow process of the discovery of new antibiotics has brought great challenges in clinical treatment and highlight the importance of developing new effective strategies for A. baumannii infections.

The innate immune system plays a critical role in the host defense against A. baumannii (Garcia-Patino et al., 2017). A better understanding of immune response to A. baumannii infection can facilitate the development of immune-adjunctive therapies. Some studies about the pathogenesis and host immune defense mechanisms against $A$. baumannii have been reported (Bruhn et al., 2015). Endothelial cells are the first line of defense against bacterial challenges (Feng et al., 2014). TLR4, mainly located on the surface of myeloid original cells such as monocytes, macrophages, microglia, myeloid DCs, and granulocytes, is the main receptor, which recognizes lipopolysaccharides (LPS) of Gram-negative bacteria, activating NF- $\kappa \mathrm{B}$ signaling pathway, leading to the production of cytokines and chemokines (Kim et al., 2013; Kawasaki and Kawai, 2014; Noto et al., 2015). Neutrophils can be recruited to the infected site by cytokines, which then engulf the pathogens recognized by the surface receptors, finally kill them through the production of reactive oxygen species (ROS) and secretion of antimicrobial peptides (van Faassen et al., 2007). Some other molecular/signaling mechanisms of infection response were also under investigation, such as neutrophil extracellular traps (NETs) (Kamoshida et al., 2015), IL-17 signaling pathway (Breslow et al., 2011), and inflammasome NLRP3 (Dikshit et al., 2018). However, most of these studies focused on specific proteins or pathways, ignoring the complicated networks of the human immune response. Hence, analyzing the global level responses will advance our understanding of host-pathogen interactions.

The rapid development of high-throughput mass spectrometry provides an effective analytical quantitative profiling platform for the systematic study of proteome changes of host response. Ferrer-Navarro et al. compared the proteome profiles of mouse lungs infected by Streptococcus pneumoniae (Gram-positive bacteria) with control mice at 24 and 48 h postinfection by 2D-differential gel electrophoresis (2D-DIGE). 91 differentially expressed proteins were identified, and the analysis showed that the cytoskeleton of host lung tissue cells is modified in S. pneumoniae infection (Ferrer-Navarro et al., 2018). Seddigh et al. (2017) applied a label-free proteome analysis of alveolar epithelial cells infected with $A$. fumigatus (fungus), which study revealed the key role of IL-41 in the host defense. Varnum et al. (2012) showed that the expression levels of numerous host proteins in bronchoalveolar lavage fluid (BALF) are altered in response to mice pulmonary infection with Francisella tularensis novicida (Gram-negative bacteria), and specific early infection biomarkers were identified. Currently, the molecular mechanisms of host-pathogen interaction in the A. baumannii

${ }^{1}$ http://www.chinets.com infected lung tissue are poorly understood and need detailed investigations. Tandem mass tag (TMT) proteomics is a widely used proteomics approach that allows high sample multiplexing and provides more accurate quantification of protein changes between samples than label-free approach by excluding some of the technical variability related to sample preparation. In the present work, 10-plex TMT-labeling quantitative proteomic by LC-MS/MS was employed to identify the differentially expressed proteins in mice lung tissue infected by A. baumannii, and bioinformatic analysis was conducted to provide proteomic evidence for the host immune process.

\section{MATERIALS AND METHODS}

\section{Bacterial Strain and Animals}

The A. baumannii strain ATCC 19606 was purchased from the American Type Culture Collection. Bacteria was cultured on a Mueller-Hinton agar (Oxoid, United Kingdom) plate and grown for $18-22 \mathrm{~h}$ at $35^{\circ} \mathrm{C}$. A single colony was selected and subcultured in Muller-Hinton broth and grown to $\mathrm{OD}_{600 \mathrm{~nm}}$ 0.2.

Specific-pathogen-free (SPF) female ICR mice (Sipper-BK, Shanghai, China), 24-26 g, were used in the lung infection model. The experiments were approved by the Experimental Animal Ethics Committee of Pharmacy, Fudan University (2019-03-HSYY-ZJ-01) and followed the Experimental Animal Welfare Review Guide.

\section{Lung Infection Model}

30 mice were randomly separated into two groups: control group (CON, $n=9$ ) and infection group (INF, $n=21$ ), and were anesthetized with an intraperitoneal injection of $25 \mathrm{mg} / \mathrm{kg}$ 2,2,2-tribromoethanol. The mice in INF group were intranasally inoculated with $50 \mu \mathrm{L}$ of $A$. baumannii suspension $\left(10^{7}\right.$ $\mathrm{CFU} / \mathrm{mL}$ ), and the $\mathrm{CON}$ group received $50 \mu \mathrm{L}$ saline solution.

For the proteomic study, both CON and INF groups had three replicates, each replicate mixed lung tissues from three different mice. Hence, 18 mice from both animal groups were sacrificed at $6 \mathrm{~h}$ after inoculation and lungs were collected and washed twice in saline and stored at $-80^{\circ} \mathrm{C}$ before protein extraction.

For the bacteria counting in lungs in the INF group, 12 mice were sacrificed at $2,6,12$, and $24 \mathrm{~h}$ after bacteria inoculation (three replicates for each time point). Specifically, lungs were removed aseptically and homogenized in $1.8 \mathrm{~mL}$ sterile saline using homogenizer (France, Bertin Technologies, Bertin Precellys 24). The homogenate was serially diluted with sterile saline and $100 \mu \mathrm{L}$ of diluent was cultured on Muller-Hinton agar at $37^{\circ} \mathrm{C}$ for $18-22 \mathrm{~h}$. The number of CFU was counted as $\log _{10}$ (CFU/lung) in lung tissue.

\section{Extraction and Trypsin Digestion of Lung Proteins}

The lung tissues were ground into cell powder in liquid nitrogen and four volumes of lysis buffer ( $8 \mathrm{M}$ urea, $1 \%$ protease inhibitor cocktail) were added to the powder, followed by sonication three times on ice using a high-intensity ultrasonic processor (Scientz). 
The remaining debris was removed by centrifugation at $12,000 \mathrm{~g}$ at $4^{\circ} \mathrm{C}$ for $10 \mathrm{~min}$. The supernatant was then collected and the protein concentration was determined with BCA kit (Beyotime) according to the manufacturer's instructions. For digestion, the protein solution was reduced with $5 \mathrm{mM}$ dithiothreitol for $30 \mathrm{~min}$ at $56^{\circ} \mathrm{C}$ and alkylated with $11 \mathrm{mM}$ iodoacetamide for $15 \mathrm{~min}$ at room temperature in darkness. The protein sample was then diluted by adding $100 \mathrm{mM}$ triethylamonium bicarbonate (TEAB) until the urea concentration reached $2 \mathrm{M}$. Finally, trypsin was added at 1:50 trypsin-to-protein mass ratio for the first digestion overnight and 1:100 trypsin-to-protein mass ratio for a second $4 \mathrm{~h}$ digestion.

\section{0-Plex TMT Labeling}

After trypsin digestion, the peptide was desalted using Strata X C18 SPE column (Phenomenex) and vacuum-dried. Peptides were reconstituted in $0.5 \mathrm{M}$ TEAB and labeled according to the manufacturer's protocol for TMT kit (Thermo). Briefly, one unit of TMT reagent was thawed and reconstituted in acetonitrile, then mixed with the peptide digest and incubated for $2 \mathrm{~h}$ at room temperature. All the labeled peptides were pooled, desalted and dried during centrifugation in vacuum before LCMS/MS analysis.

\section{LC-MS/MS Analysis}

The mixed 10-plex TMT-labeled tryptic peptides were fractionated using reverse-phase HPLC with an Agilent 300 Extend C18 column (5 $\mu \mathrm{m}, 4.6 \mathrm{~mm}$ ID, $250 \mathrm{~mm}$ length). The peptides were separated with a gradient of $8-32 \%$ acetonitrile ( $\mathrm{pH} 9.0$ ) over $60 \mathrm{~min}$ into 60 fractions and combined into 18 fractions and dried by vacuum centrifugation. The fractionated peptides were redissolved in $0.1 \%$ formic acid (solvent A) and injected into Q Exactive ${ }^{\mathrm{TM}}$ Plus (Thermo) coupled online to an EASY-nLC 1000 UPLC platform for the proteomic analysis. The peptides were separated by a home-made reversed-phase analytical column (15 cm length, $75 \mu \mathrm{m}$ i.d.). The gradient was started with an increase of solvent B from 5 to $22 \%$ ( $0.1 \%$ formic acid in $98 \%$ acetonitrile) over $40 \mathrm{~min}$, continued increasing from 22 to $35 \%$ in $13 \mathrm{~min}$, and finally increased to $80 \%$ in $3 \mathrm{~min}$ and held for $4 \mathrm{~min}$. The flow rate was constant set as $800 \mathrm{~nL} / \mathrm{min}$ and the solvent A consisted of $0.1 \%$ formic acid aqueous solution.

A data-dependent acquisition (DDA) consisted of duty cycle starting with one single-stage MS scan and followed by $20 \mathrm{MS} / \mathrm{MS}$ scans with $15.0 \mathrm{~s}$ of dynamic exclusion time of a fragmented precursor m/z. Automatic gain control (AGC) was set at $5 \times 10^{4}$. MS1 spectra were collected in the range of $350-1,800 \mathrm{~m} / \mathrm{z}$ at a resolution of 70,000, and MS2 spectra were collected at a resolution of 17,500 and the fixed first mass was set as $100 \mathrm{~m} / \mathrm{z}$. The isolation width for MS2 scan was $2.0 \mathrm{Da}$. The collision energy was set as $28 \%$.

\section{Database Search and Statistical Analysis}

The MS/MS spectra data were processed using MaxQuant (v.1.5.2.8). Tandem mass spectra were searched against the SwissProt Mouse database (29,795 sequences) concatenated with reverse decoy database. Trypsin/P was specified as cleavage enzyme allowing up to 2 missed cleavages. The mass tolerance for precursor ions was set as $20 \mathrm{ppm}$ in the first search and $5 \mathrm{ppm}$ in the main search, and the mass tolerance for fragment ions was set as $0.02 \mathrm{Da}$. Carbamidomethyl on Cys was set as fixed modification and oxidation on Met residue was specified as variable modifications. FDR was adjusted to $<1 \%$ at PSM, peptide and protein levels and the minimum score for peptides was set $>40$. The correction for isotope cross contamination has been performed in MaxQuant based on data provided by Thermo Scientific.

Statistical analysis was performed with the $t$-test using $\mathrm{R}$ software version 3.5.1. Given that the fold change compression of TMT has made the observed differences smaller compared to the real differences, a cutoff of the nominal $p<0.05$ was considered to be significant and the fold change $>1.2$ or $<0.83$ was required to identify proteins differentially expressed.

\section{Bioinformatics Analysis}

The STRING Database ${ }^{2}$ and gProfiler $^{3}$ were used to analyze the differentially expressed proteins for Gene Ontology (GO) annotation, KEGG and Reactome pathway analysis. A dataset of all the differentially expressed protein identifiers was uploaded to STRING and Mus musculus was chosen as organism classification. The minimum required interaction score was 0.4 for each protein-protein association.

\section{Validation}

Lung of six mice were used for Elisa validation. Three mice in control group and the other three in infection group. The MMP-9 Elisa kit (R\&D, MMPT90, the detection range was $0.08 \sim 5 \mathrm{pg} / \mathrm{mL}$ ) and S100a8/a9 Elisa kit (R\&D, DY859605 , the detection range was $125 \sim 5,000 \mathrm{pg} / \mathrm{mL}$ ) were used according to the manufacturer's instructions to determine the concentration of proteins in the tissue homogenate. The proinflammatory factor IL-6 (R\&D, DY406-05, the detection range was $15.6 \sim 1,000 \mathrm{pg} / \mathrm{mL}$ ) was also determined to show the host inflammatory response.

\section{RESULTS}

\section{Bacteria Counting After Infection}

The scheme of the experimental design is shown in Figure $\mathbf{1}$. Mice were randomly separated into two categories: the proteomic and bacteria counting categories. 18 mice were designated into proteomic category and separated into control group (CON, $n=9$ ) and infection group (INF, $n=9$ ). Lung tissues were collected from each mice and pooled to prepare three replicate samples, using three animals per pool. The rest of mice $(n=12)$ were in the bacteria counting category, in which they were infected with bacteria and sacrificed at 2, 6,12 , and $24 \mathrm{~h}$ after bacteria inoculation (three replicates for each time point). Bacterial load at the lung, $2 \mathrm{~h}$ postinfection, was $6.29 \pm 0.05 \log _{10}$ CFU/lung tissue after

\footnotetext{
${ }^{2}$ www.string-db.org

${ }^{3}$ https://biit.cs.ut.ee/gprofiler/gost
} 


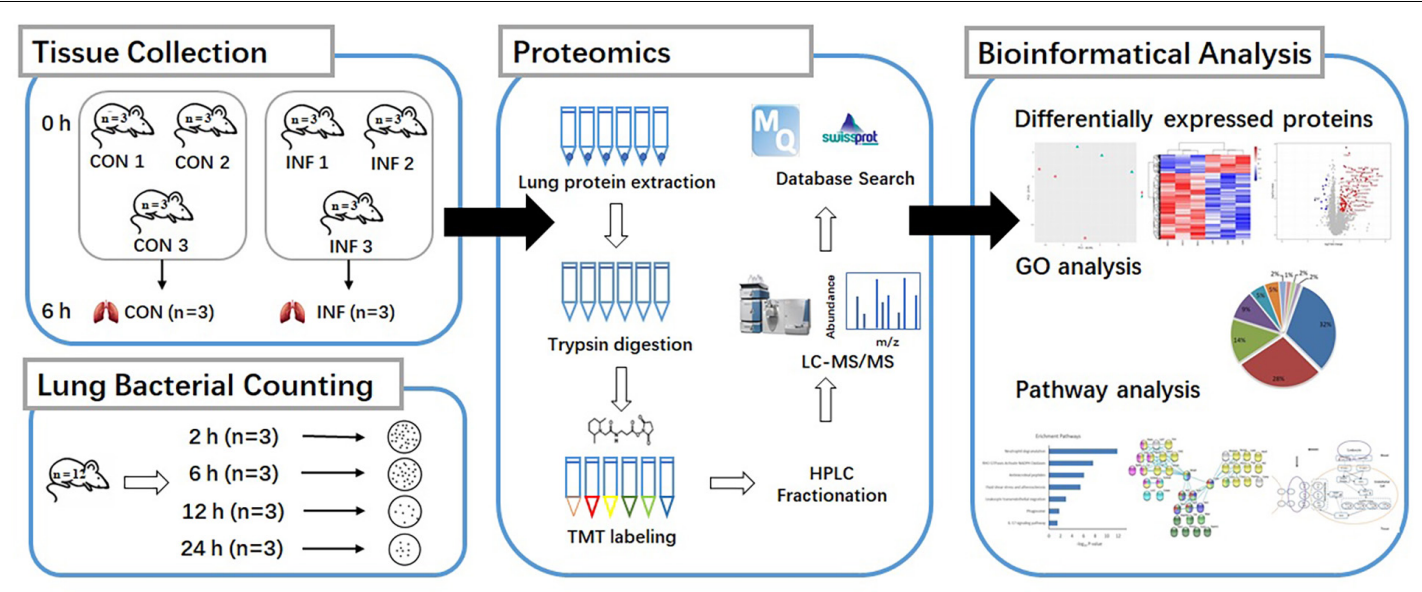

FIGURE 1 | Experimental setup, sample preparation and bioinformatic workflow for comparison proteome of $A$. baumannii infected mice lung tissue (INF) with the control group (CON).

intranasally infected with $1 \times 10^{5} \mathrm{CFU} /$ lung (5.7 $\log _{10} \mathrm{CFU} / \mathrm{lung}$ ) A. baumannii. It remained basically unchanged at $6 \mathrm{~h}(5.97 \pm 0.12$ $\log _{10}$ CFU/lung). However, the bacterial counts at $12-24 \mathrm{~h}$ decreased to $3.13 \pm 0.23$ and $3.28 \pm 0.34 \log _{10}$ CFU/lung, respectively (Figure 2).

\section{Proteomic Expression Patterns in A. baumannii-Infected Mice}

Overall, 6,218 proteins were identified in the two groups (CON and INF) in which 6,172 could be quantified with reporter ion intensity detected in at least one MS/MS spectra. As shown in the principal component analysis (PCA) score plots and heatmap, the A. baumannii infection induced significant proteome changes $6 \mathrm{~h}$ after inoculation (Figures 3A,B). Proteins with relative expression level fold change $>1.2$ or $<0.83$ were considered as differentially expressed proteins (DEPs, $p<0.05$ ). The volcano plots for the DEPs are shown in Figure 3C. There were 121 DEPs between the INF and CON mice groups, with 108 upregulated and 13 down-regulated proteins in the INF group. The full list of differentially expressed proteins identified between CON and INF sample groups of 18 mice lung can be found in Supplementary Table 1.

\section{Functional Characterization of Differentially Expressed Proteins}

Based on GO database, the DEPs were classified into different biological process, cellular component and molecular function (Figures 4A-C). The DEPs were mainly located in cytoplasm, intracellular organelle, membrane, and extracellular region, and they were mainly involved in the molecular function of ion binding, lipid binding and serine-type endopeptidase activity. For biological process, the DEPs were involved in response to stress, transport, immune system process and programmed cell death. The most important GO categories included cytokine production (GO.0001816, CD14, Camp, Mmp8, Pglyrp1, Elane),

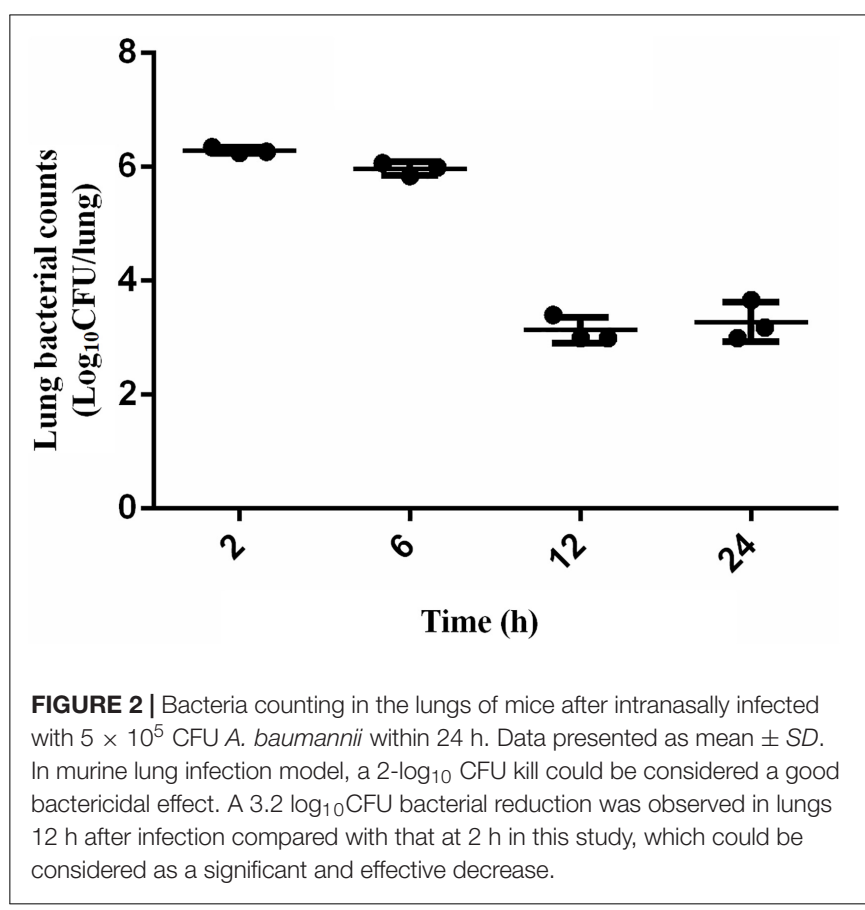

antimicrobial humoral defense (GO.0019730, Camp, Ltf, Sftpd, Ctsg), NADPH oxidase complex (GO.0043020, Cybb, Ncf1, Ncf2, Ncf4), and apoptotic process (GO.0006915, S100a8, S100a9, Lcn2, Hmox1).

KEGG pathway analysis of DEPs revealed that multiple immune-related pathways are involved in the host responses including fluid shear stress and atherosclerosis, leukocyte transendothelial migration, phagocytosis, and IL-17 signaling pathways. A search using the Reactome database revealed DEPs participating in signaling pathways such as neutrophil degranulation, RHO GTPases Activate NADPH oxidases and antimicrobial peptides (Figure 4D). STRING protein-protein interaction database was used to further explore the interactions 

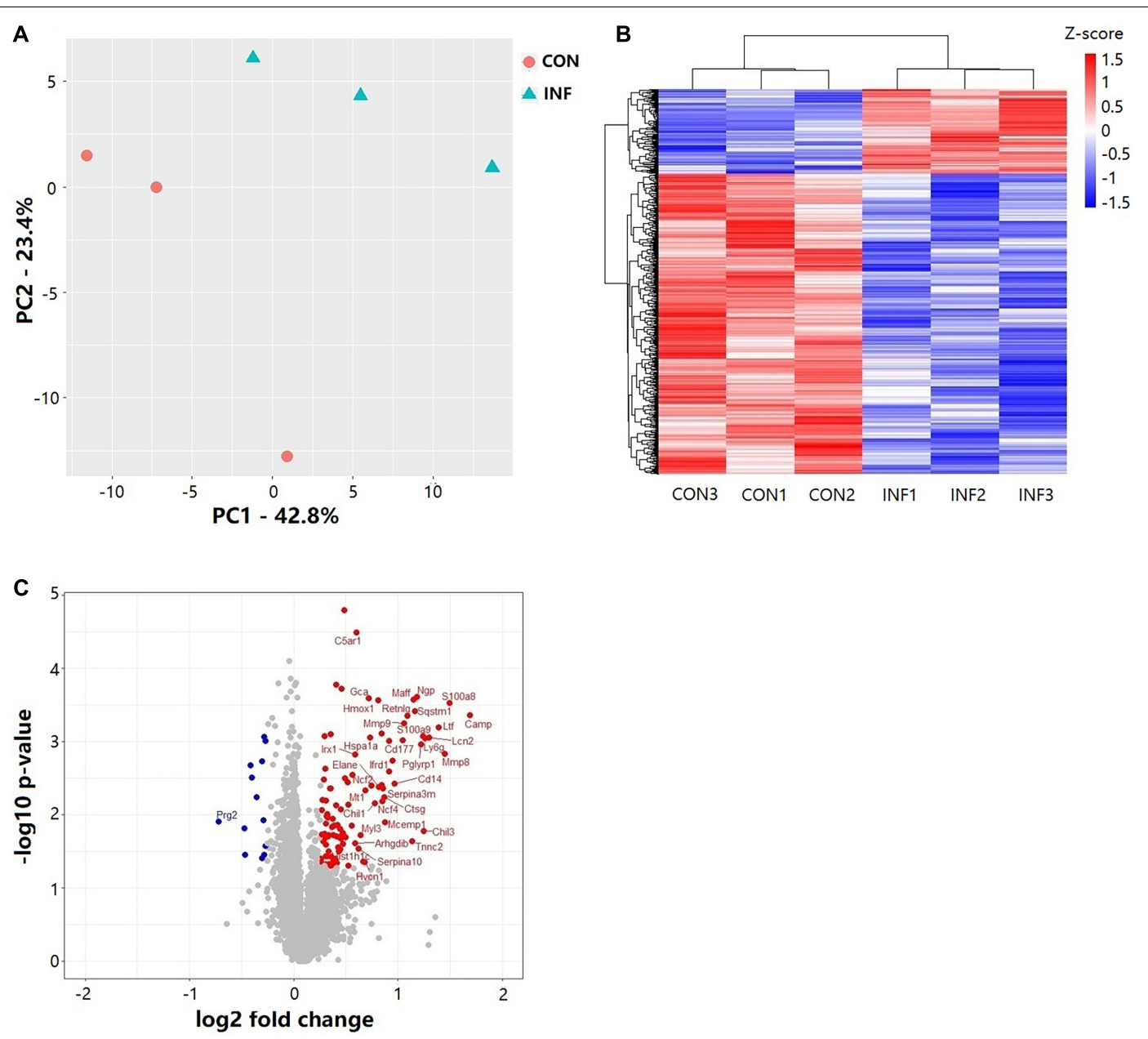

FIGURE 3 | Results of TMT label quantitative proteome analysis of mice lungs. (A) PCA score plots of the first two principal components of proteomic expression levels in mice lungs of CON and INF groups. Each group has 3 biological replicates, each replicate composes of three different mice. (B) Hierarchical clustering of proteins with Student's $t$-test with nominal $p \leq 0.05$. (C) Volcano plot showing the differential proteins up and down regulated in INF group. Student's $t$-test nominal $p \leq 0.05$ and a ratio of means $\geq 1.2$ or $\leq 0.83$ are applied as significance thresholds. Differentially expressed proteins are highlighted in red (up-regulated) or blue (down-regulated). The gene names are given for the proteins whose ratio $\geq 1.5$ or $\leq 0.67$.

between proteins in significantly enriched pathways. STRING analysis showed that the proteins were divided into three main protein-protein interaction clusters. Figure $4 \mathrm{E}$ shows that the tight protein-protein clusters reflected consistently enriched pathways, except for neutrophil degranulation, which was present in multiple clusters, which is possibly due to diverse functions of neutrophil secreted proteins. Multiple proteins participated in more than one pathways, especially Mmp9, Cybb, Ncf1, Ncf2, Ncf4, and Rac2. It's worth mention that both Cybb and Mmp9 participate in four pathway and link together multiple clusters, which suggested their important role in the lung response to A. baumannii infection. The 38 DEPs (Table 1) part of key pathways are shown in Figures 4DF including neutrophil degranulation, RHO GTPases Activate NADPH oxidases and antimicrobial peptides. The details of the GO, KEGG and REACTOME pathways of all the differentially expressed proteins between INF and CON groups were shown in Supplementary Table 2 .

\section{Validation}

The validation using Elisa kits found that key proteins Mmp9 and S100a8/a9 were significantly increased in mouse lung tissue during A. baumannii clearance (Infection versus Control, nominal $p<0.05$, Figures $5 \mathbf{A}, \mathbf{B})$. This result is in agreement with the protein levels measured by TMT analysis. The expression level of IL- 6 was higher at $6 \mathrm{~h}$ after infection than that in control mice, although with no significant difference (Infection versus Control, nominal $p=0.10$, Figure $5 \mathrm{C}$ ). It also suggested the occurrence of immune response.

\section{DISCUSSION}

A. baumannii is one of the major pathogens endangering public health where the host immune system plays as an important barrier, and the infection usually occurs when the host is immunocompromised. In the treatment of $A$. baumannii 


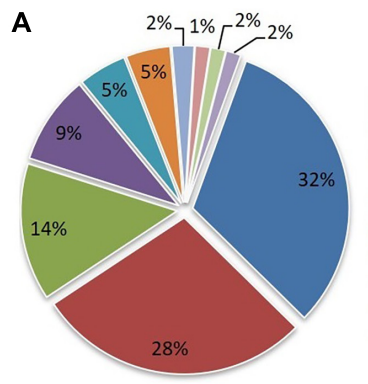

- intracellular organelle

- cytoplasmic part

- extracellular region

- cytoplasmic vesicle

m secretory vesicle

In secretory granule

- rough endoplasmic reticulum

- NADPH oxidase complex

- lipoprotein particle

= protein-lipid complex

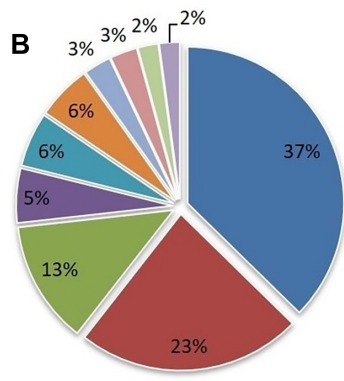

- ion binding

- anion binding

= lipid binding

- serine-type endopeptidase activity

- glycosaminoglycan binding

= sulfur compound binding

$=$ glucose binding

- lipopolysaccharide binding

w super oxide-generating NADPH oxidase

activator activity

activator activity
moxıdoreductase activity, actıng on

$\mathrm{NAD}(\mathrm{P}) \mathrm{H}$, oxygen as acceptor

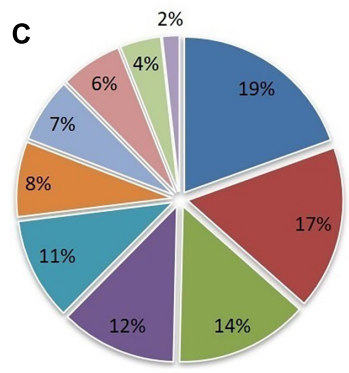

II reactive oxygen species metabolic process

= neutrophil mediated immunity
D

Erichment Pathways

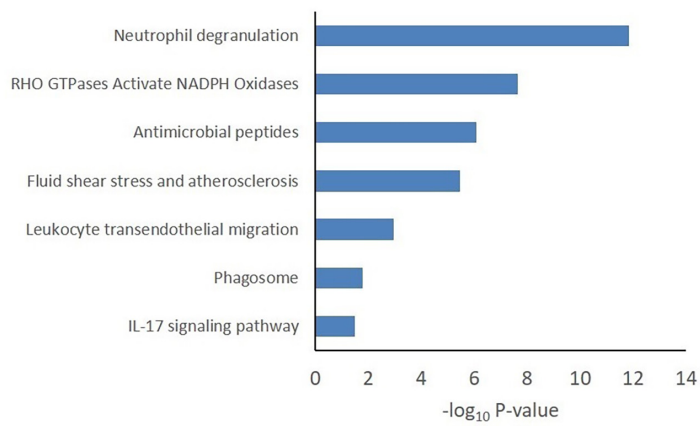

E

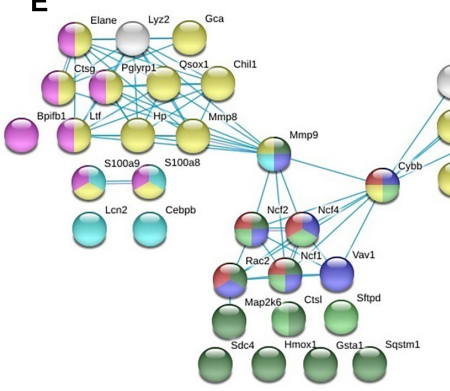

Fluid shear stress and atherosclerosis

Leukocyte transendothelial migration

Phagosome

IL-17 signaling pathway

Neutrophil degranulation

Antimicrobial peptides

RHO GTPases Activate NADPH Oxidases

FIGURE 4 | GO, enrichment pathways and protein-protein interactions of DEPs. (A) Cellular component (GO analysis); (B) molecular function (GO analysis); (C) biological process (GO analysis); (D) pathways (KEGG and REACTOME); (E) protein-protein interaction using STRING database; (F) expression of proteins involved in key pathways.
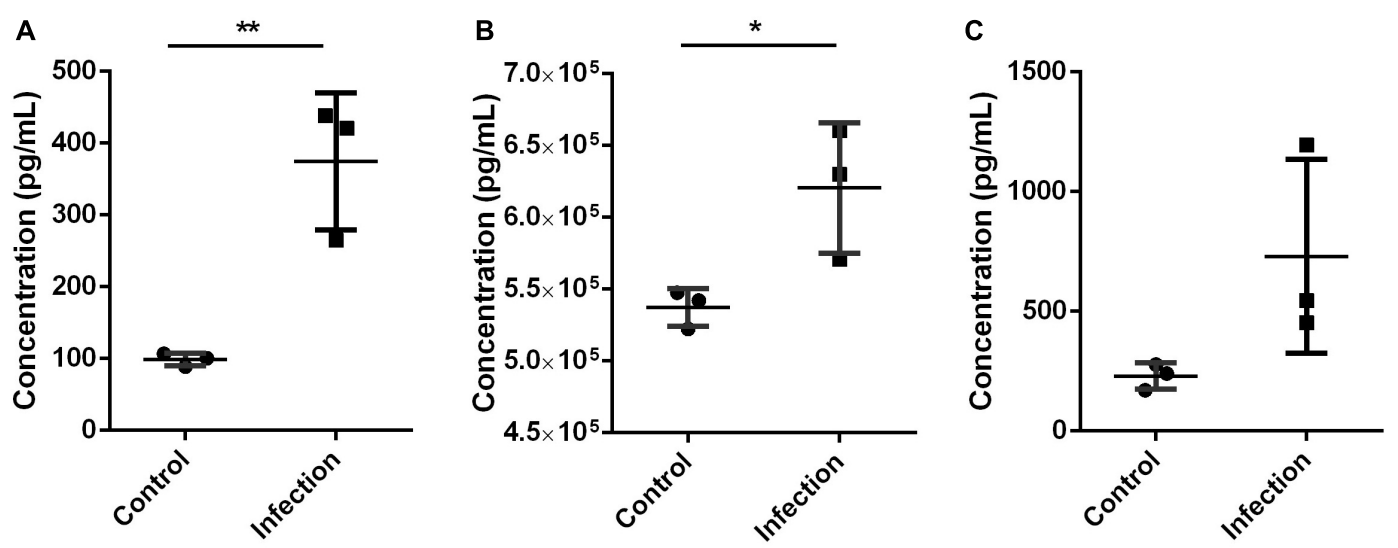

FIGURE 5 | Expression level of Mmp9 (A), S100a8/a9 (B), and IL-6 (C) in mice lung tissue $6 \mathrm{~h}$ after inoculated with A. baumannii by Elisa. MMP9 and S100a8/a9 showed the same expression pattern with proteomics analysis. Values represent the means \pm SD of three replicates. Shapiro-Wilk test was performed on the Elisa data that showed the data followed a normal distribution $(p>0.05)$, so $t$-test was employed to compare the Elisa data between the two groups. Student's $t$-test, ${ }^{*} p<0.05 ;{ }^{* *} p<0.01$. 
TABLE 1 | DEPs involved in key pathways.

\begin{tabular}{|c|c|c|c|c|}
\hline Uniprot ID & Gene name & Protein name & Expression fold change & $-\log _{10} p$-value \\
\hline P30993 & C5ar1 & Complement component 5a receptor 1 & 1.52 & 4.49 \\
\hline P48999 & Alox5 & Arachidonate 5-lipoxygenase & 1.38 & 3.72 \\
\hline Q8VC88 & Gca & Grancalcin & 1.65 & 3.59 \\
\hline P27005 & S100a8 & S100 calcium binding protein A8 (calgranulin A) & 2.82 & 3.53 \\
\hline Q64337 & Sqstm1 & Sequestosome 1 & 2.23 & 3.41 \\
\hline P51437 & Camp & Cathelicidin antimicrobial peptide & 3.23 & 3.36 \\
\hline P41245 & Mmp9 & Matrix metallopeptidase 9 & 2.08 & 3.25 \\
\hline P08071 & Ltf & Lactotransferrin & 2.62 & 3.19 \\
\hline P31725 & S100a9 & S100 calcium binding protein A9 (calgranulin B) & 2.36 & 3.07 \\
\hline Q61696 & Hspa1a & Heat shock protein $1 \mathrm{~A}$ & 1.66 & 3.06 \\
\hline P11672 & Lcn2 & Lipocalin 2 & 2.46 & 3.05 \\
\hline Q8R2S8 & Cd177 & CD177 antigen & 2.07 & 3.02 \\
\hline Q61114 & Bpifb1 & BPI fold containing family $\mathrm{B}$, member 1 & 6.28 & 2.96 \\
\hline O88593 & Pglyrp1 & Peptidoglycan recognition protein 1 & 2.34 & 2.96 \\
\hline 070138 & Mmp8 & Matrix metallopeptidase 8 & 2.73 & 2.83 \\
\hline P05555 & Itgam & Integrin subunit alpha M & 1.93 & 2.74 \\
\hline P11835 & Itgb2 & Integrin subunit beta 2 & 1.47 & 2.54 \\
\hline Q61093 & Cybb & Cytochrome b-245, beta polypeptide & 1.43 & 2.44 \\
\hline P10810 & Cd14 & CD14 antigen & 1.95 & 2.43 \\
\hline 070145 & Ncf2 & Neutrophil cytosolic factor 2 & 1.67 & 2.40 \\
\hline Q3UP87 & Elane & Elastase, neutrophil expressed & 1.76 & 2.38 \\
\hline Q03734 & Serpina3m & Serine (or cysteine) peptidase inhibitor, clade A, member 3M & 1.81 & 2.36 \\
\hline Q61646 & $\mathrm{Hp}$ & Haptoglobin & 1.27 & 2.36 \\
\hline P28293 & Ctsg & Cathepsin G & 1.83 & 2.24 \\
\hline P06797 & Ctsl & Cathepsin L & 1.21 & 2.20 \\
\hline P97369 & Ncf4 & Neutrophil cytosolic factor 4 & 1.80 & 2.19 \\
\hline Q61362 & Chil1 & Chitinase-like 1 & 1.71 & 2.16 \\
\hline Q3TRM8 & Hk3 & Hexokinase 3 & 1.43 & 2.13 \\
\hline P70236 & Map2k6 & Mitogen-activated protein kinase kinase 6 & 0.82 & 1.93 \\
\hline Q61878 & Prg2 & Proteoglycan 2, bone marrow & 0.61 & 1.91 \\
\hline Q09014 & Ncf1 & Neutrophil cytosolic factor 1 & 1.30 & 1.72 \\
\hline Q05144 & Rac2 & Rac family small GTPase 2 & 1.41 & 1.69 \\
\hline P27870 & Vav1 & Vav 1 oncogene & 1.22 & 1.66 \\
\hline P50404 & Sftpd & Surfactant associated protein D & 1.35 & 1.56 \\
\hline P32037 & Slc2a3 & Solute carrier family 2 (facilitated glucose transporter), member 3 & 1.36 & 1.53 \\
\hline Q8BND5 & Qsox1 & Quiescin Q6 sulfhydryl oxidase 1 & 1.29 & 1.42 \\
\hline Q3U2S8 & Hven1 & Hydrogen voltage-gated channel 1 & 1.60 & 1.35 \\
\hline P28033 & Cebpb & CCAAT/enhancer binding protein (C/EBP), beta & 1.26 & 1.32 \\
\hline
\end{tabular}

infection, the development of drug resistance leads to an important lack of clinically selectable drugs, and immune-related treatment regimens enforcing host immune response are taken into consideration. The mechanism of the host response to A. baumannii has not been elucidated. In this study, we firstly described the proteome changes of mice lung tissue during the clearance of A. baumannii, and analyzed their functions as well as involved pathways.

Bacterial counts in the lung tissue of mice showed a decrease of bacteria between 6 and $12 \mathrm{~h}$ after infection, and the up-regulation of a large number of immune-related proteins demonstrated the immune response. The increased level of IL-6 also suggested the immune response, although with no significant difference possibly due to the inappropriate sampling time. We observed a significant up-regulation of proteins involved in receptor recognition (CD14, S100a8, S100a9, Itgb2, Itgam). CD14 is a GPI-anchored cell surface molecule that functions as a receptor for LPS, the major cell membrane component of Gram-negative bacteria, and upon contact with the bacterial surface transfers the subsequent molecular response to eliminate the pathogens. The binding of LPS to CD14 plays a key role in innate immune against $A$. baumannii via LPS moiety which helped to effectively eliminate the bacteria from lungs (Knapp et al., 2006; Park and Lee, 2013). S100a8 and S100a9 are subunits of an antimicrobial heterodimer, calprotectin. Imaging MS showed a strong correlation between calprotectin expression and bacterial burden in A. baumannii pneumonia mice, which can be used as a marker of inflammatory response (Moore et al., 2014). 


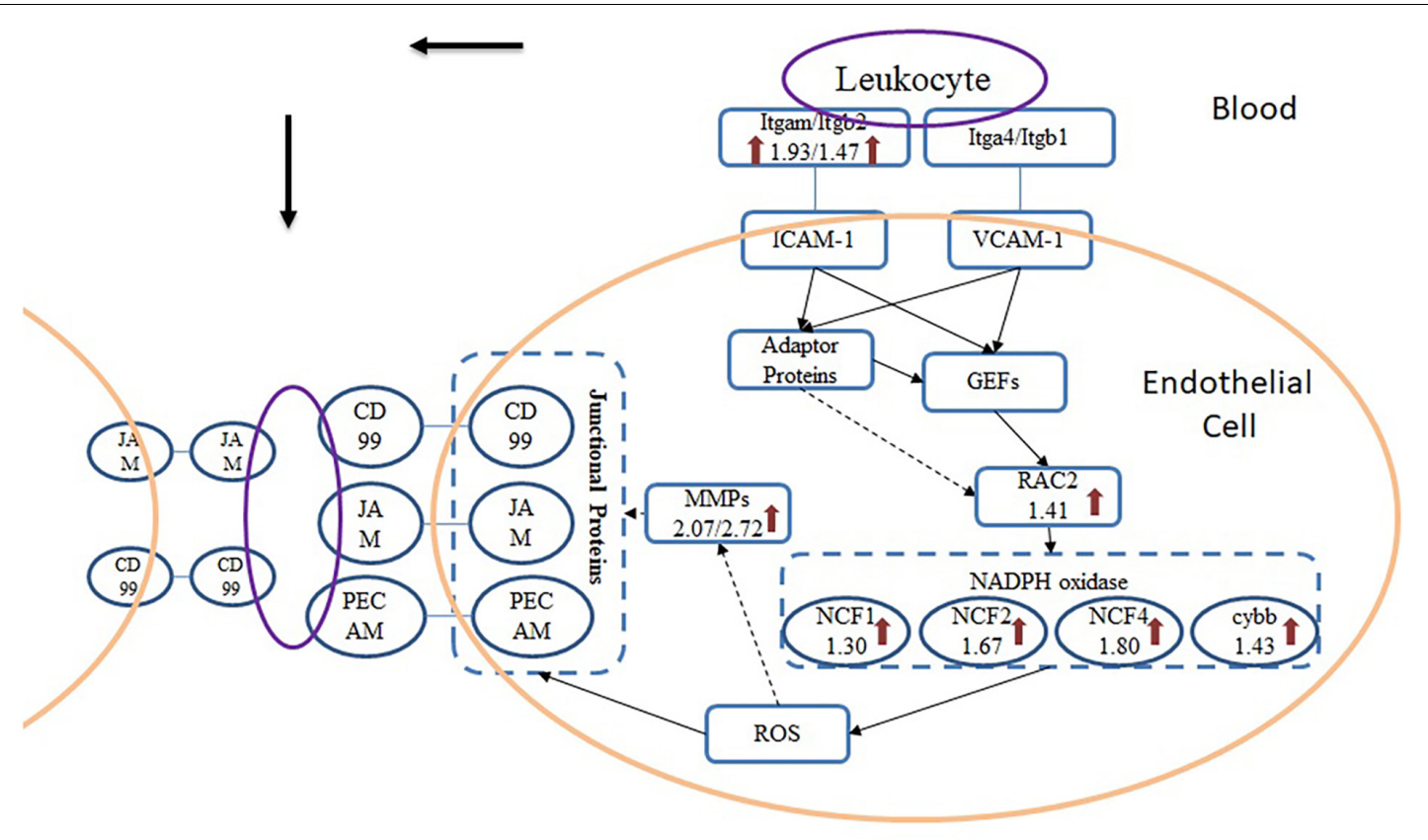

Tissue

FIGURE 6 | Scheme of TEM pathway during A. baumannii infection. Arrows indicate the proteins that were significantly up-regulated and the numbers beneath the gene names represent fold change.

S100A9-/- mice had higher mortality and bacterial burden compared with the wildtype after infected with A. baumannii. The reason of this higher mortality can be because calprotectin could inhibit A. baumannii growth through the chelation of $\mathrm{Mn}$ and $\mathrm{Zn}$. This calprotectin-meditated $\mathrm{Zn}$ sequestration also participates in the A. baumannii drug resistance development through the influence on $\mathrm{Zn}$ acquisition system (Hood et al., 2012). Besides, S100a9 was indicated that it can bind CD14 functioning as a co-receptor for the S100a9-mediated TLR4signaling pathway (Haziot et al., 2016). Igtam/Itgb2, also known as CD11b/CD18, Mac-1 or CR-3, is a surface receptor integrin expressed on many innate immune cells including monocytes, granulocytes and macrophages (Hughes et al., 1992; Zhou et al., 2013). A. baumannii inhibits neutrophil-induced NET formation by suppressing the adhesion ability of neutrophils, partly due to suppression CD11a expression but not of the CD11b expression on neutrophil surface (Kamoshida et al., 2018). Our study similarly detected an increased expression of CD11b, indicating that $\mathrm{CD} 11 \mathrm{~b}$ is not associated with the decreased adhesion in NET formation.

Proteins involved in anti-microbial defense (Camp, Ltf, Ctsg, Sftpd) were also up-regulated during the bacteria clearance process. Cathelicidin antimicrobial peptide (Camp) serves as a component of innate response and they are mainly stored in neutrophil and macrophage granules. The amphophilic property of Camp enables its accurate adhesion and anchoring, embedding in the lipid bilayer and creating transmembrane pores (Kosciuczuk et al., 2012), which leads to bacteria-killing. Recently, it has been shown that a variety of antimicrobial peptides have good antibacterial activity against multi-drug resistant A. baumannii and can be used as a potential therapy (Barksdale et al., 2017; Liu et al., 2017; Spencer et al., 2018). Lactotransferrin (Ltf) widely occurs in the blood and biological fluid. Upon inflammatory stimulates, Ltf can be recruited and delivered at the inflammatory site by neutrophils and released in iron-free form so that its anti-microbial activity can be activated through iron scavenging property. Colistin-induced LPS-deficient $A$. baumannii presented with increased sensitivities to lysozyme and lactoferrin in vitro (Kamoshida et al., 2020), and the combination of lactoperoxidase and lactoferrin also showed antibacterial activity in mice with $A$. baumannii pneumonia (Mahdi et al., 2018). Cathepsin G (Ctsg) is a serine protease that is involved in direct intracellular killing process of phagocytosed bacteria with myeloperoxidase (MPO) and ROS. Besides, it participates in extracellular killing of bacteria by modifying DNA structure of the pathogen, which serves as a component of NETs. Neutrophil-derived Ctsg critically contributes to the deceleration of pathogen replication during the mycobacterial lung infection of mice (Steinwede et al., 2012), and Ctsg can be potently inhibited by Staphylococcus aureus immune evasion proteins (Herdendorf et al., 2020). There are few reported studies relevant to the Ctsg effect on A. baumannii. Surfactant protein D (Sftpd) is reported to be an important component of the immune system of the lung. It can recognize LPS of Gram-negative bacteria, leading to the formation of CRD (Carbohydrate recognition domain)-dependent bacterial aggregation which enhances the binding to its receptors such as CD14 (Crouch and Wright, 2001). This binding process provides a potential treatment of 
bacterial infection diseases (Reinhardt et al., 2016). We found these proteins up-regulated indicating their important roles in the clearance of $A$. baumannii from mouse lung tissue. Ten abundant proteins involved in Antimicrobial peptides pathway (REAC:R-MMU-6803157), suggesting that antimicrobial peptide is a key killing mechanisms of A. baumannii.

From the results of pathway analysis, we found that Leukocyte Transendothelial Migration (TEM) and Phagosome pathway were activated. TEM refers to the process that leukocyte crosses blood vessel barrier from blood to the inflammatory site. After infected with A. baumannii strain ATCC19606, eight proteins in the lung of mice involved in TEM were significantly up-regulated (Figure 6), and they were related to multistep function of TEM pathway including recognition, adhesion, signal transduction in endothelial cells and transmigration. TEM is currently regarded as a multistep process including leukocyte capture, firm adhesion, crawling and transmigration in paracellular and transcellular routes, modulated by inflammatory cytokines and intracellular signaling events (Nourshargh et al., 2010; Vestweber, 2010; Muller, 2011; Schimmel et al., 2017). The initial adhesion of leukocyte and endothelial cell (EC) dependent on the interaction between P- or E-selectin on ECs and their ligands, which induces the cluster of EC receptors including ICAM-1 and VCAM-1 (Langer and Chavakis, 2009). In the TEM process, Itgam expressed on the surface of leukocyte serves as a ligand interacting with ICAM-1 on the endothelial cells. This binding induces the recruitment and phosphorylation of actin-binding adaptor proteins such as cortactin, leading to actin polymerization and recruitment of more ICAM-1 to the site of leukocyte adhesion (Nourshargh et al., 2010). The cluster of ICAM-1 protein on endothelial cell surface also induces the intracellular release of $\mathrm{Ca}^{2+}$ and activation of GEF (Guanine nucleotide exchange factors) which drives activation of Rho family proteins including RhoA, Rac and Rho GTPase. Rac serves as the activator for NADPH oxidase (NOX), producing ROS that can induce the reduction of cell-cell contact on ECs and support the direct killing of ingested pathogens. Rac protein subfamily includes Rac1, Rac2, and Rac3 in which Rac1 and Rac2 were mostly studied. Neutrophils from Rac2 deficient mice while express normal levels of Racl demonstrate deficits in multiple functions including superoxide production (Roberts et al., 1999). We found only Rac2 significantly up-regulated in A. baumannii infected mice lung without change in Rac1 level, which also indicates that Rac2 is the predominant Rac species involved in the innate immune response rather than Rac1, especially for A. baumannii infection. ROS is generated by the activation of NOX, which subsequently passes the signaling to endothelial cells that leads to the loosening of adhesion junctions and opening of transient channels across ECs for leukocyte transcellular migration. Junctional proteins including JAM, PECAM-1 and CD99 are activated by ROS during infection and bind the leukocyte ligands such as MAC-1, leading to vascular permeability (Muller, 2011). There were no significant changes of junctional proteins in lung tissue after A. baumannii infection, but MAC-1, the ligand for JAM, is increased in abundance. Besides, we found an obvious change of MMP-9 which is activated by ROS and have the function to cleave the endothelial cell junctions (Vermeer et al., 2009), leading to the weakening of cell-cell adhesion and opens a channel through epithelia which leukocyte can migrate.

Phagocytosis occurs in specialized cells such as macrophages, neutrophils and dendritic cells which functions as a critical process of bacteria-killing and clearance of cell debris. This process is initiated by the recognition and binding of the pathogen by the phagocyte surface, which triggers the cytoskeletal reorganization, leading to the uptake of pathogen and formation of phagosome (Aderem, 2003). After encapsulated in phagosomes, the cell kills pathogens using ROS, nitrogen oxide (NO) or antibacterial peptides released from the granules. Different from neutrophils, macrophages kill pathogens through the synthesis of $\mathrm{NO}$ with cytostatic and cytotoxic activity against bacteria (Dale et al., 2008). Drug resistance may affect bacterial susceptibility to phagocytosis. Sato et al. demonstrated renewed virulence characteristics in multi-drug resistant A. baumannii after phagocytosis by macrophages, and further studies are required to understand the mechanisms (Sato et al., 2019).

We found that TEM and Phagocytosis pathways had some shared up-regulated proteins such as Ncf1, Ncf2, Ncf4, Cybb. Reactome pathway analysis suggested that these proteins were involved in RHO GTPases Activate NADPH Oxidases pathway RHO GTPases (REAC:R-MMU-5668599). NOX is a multicomponent enzyme that catalyzes the generation of $\mathrm{O}^{2-}$ from oxygen and NADPH. The four essential proteins of NOX subunit were all found significantly up-regulated in A. baumannii infected mice lung compared with the control group which demonstrates its critical role in the innate immune response of mice against $A$. baumannii lung infection. Cytochrom b588 composing of $\mathrm{gp}^{91 P H O X}$ (Cybb) and $\mathrm{p}^{22 P H O X}$ (Cyba) serves as the electron transporter. Cytosolic component $\mathrm{p}^{47 P H O X}(\mathrm{Ncf} 1)$ serves as an adaptor protein, providing a platform for the assembly of cytochrome b588, mediating the interaction both with b588 and $\mathrm{p}^{67 P H O X}$. Protein $\mathrm{p}^{67 P H O X}$ (Ncf2) is another cytosolic component containing two SH3 domains and a NADPH binding domain, which is thought to regulate the transfer electrons from NADPH to FAD. Small GTPase Rac2 also participates in electron transfer (Hordijk, 2006). The fourth component $\mathrm{p}^{40 P H O X}(\mathrm{Ncf} 4)$ contains a PX domain that may bind to special phosphoinositides, thus mediating the assembly of NOX in plasma and cell membrane (Babior et al., 2002). Qiu et al. found that $\mathrm{gp}^{91 p h o x-/-}$ mice had higher susceptibility to intranasal A. baumannii infection than wild-type C57BL/6 mice, with significantly greater bacterial counts in lungs and spleen. It is showed that NOX appears to play a crucial role in host defense against A. baumannii (Qiu et al., 2009). Our results similarly indicate that in the host response against A. baumannii, NOX is the primary part activated in TEM and Phagocytosis pathways and the NOX-dependent killing mechanism plays an important role.

To observe the bacteria clearance process by host immunity, we used immunocompetent mice in our study. But the immune response is probably different when the host is in a pathogenesis status, a hypervirulent $A$. baumannii strain or immunocompromised mouse could be used to obtain an 
infection model in a pathogenesis condition, which is closer to a human infection situation in hospitals. Comparing the host response against $A$. baumannii infection under normal and pathogenic status helps to reveal the key factors of the immune system or pathogenesis mechanisms.

In this study, multiple testing was not performed because it was found too conservative for our data resulting in too few candidate proteins. This probably because of the ratio compression of isobaric quantification (Karp et al., 2010). The moderate immune response caused by ATCC19606 and the sampling time could also be the reasons. To validate the upregulation of candidate proteins Mmp9 and S100a8/a9, we performed Elisa experiments which showed consistent results with the proteomics data. In our future study, high virulence or high bacterial inoculum will be used to observe a more drastic immune response, and the kinetic process of immune response will be studied to determine the effect time of candidate proteins.

In conclusion, in the present study, we used a TMT-labeling quantitative proteomic approach to investigate lung response of mice during bacteria clearance against $A$. baumannii. It is clear that the expression of numerous lung tissue proteins of mice was altered, especially proteins associated with receptor recognition, NADPH oxidase function and antimicrobial peptides, as well as some innate immune pathways including leukocyte transendothelial migration and phagosome. Our results provide a basis for the further study of host-organism interaction and hopefully support the development of treatment strategies of A. baumannii human lung infection.

\section{DATA AVAILABILITY STATEMENT}

The mass spectrometry proteomics data have been deposited to the ProteomeXchange with the data set identifier PXD060240.

\section{REFERENCES}

Aderem, A. (2003). Phagocytosis and the inflammatory response. J. Infect. Dis. 187 (Suppl. 2):S340. doi: 10.1086/374747

Babior, B. M., Lambeth, J. D., and Nauseef, W. (2002). The neutrophil NADPH oxidase. Arch. Biochem. Biophys. 397, 342-344. doi: 10.1006/abbi.2001.2642

Barksdale, S. M., Hrifko, E. J., and Van Hoek, M. L. (2017). Cathelicidin antimicrobial peptide from Alligator mississippiensis has antibacterial activity against multi-drug resistant Acinetobacter baumanii and Klebsiella pneumoniae. Dev. Comp. Immunol. 70, 135-144. doi: 10.1016/j.dci.2017.01.011

Breslow, J. M., Meissler, J. J. Jr., Hartzell, R. R., Spence, P. B., Truant, A., Gaughan, J., et al. (2011). Innate immune responses to systemic Acinetobacter baumannii infection in mice: neutrophils, but not interleukin-17, mediate host resistance. Infect. Immun. 79, 3317-3327. doi: 10.1128/IAI.00069-11

Bruhn, K. W., Pantapalangkoor, P., Nielsen, T., Tan, B., Junus, J., Hujer, K. M., et al. (2015). Host fate is rapidly determined by innate effector-microbial interactions during Acinetobacter baumannii bacteremia. J. Infect. Dis. 211, 1296-1305. doi: 10.1093/infdis/jiu593

Crouch, E., and Wright, J. (2001). Surfactant proteins a and d and pulmonary host defense. Annu. Rev. Physiol. 63, 521-554. doi: 10.1 146/annurev.physiol.63.1.521

Dale, D. C., Boxer, L., and Liles, W. C. (2008). The phagocytes: neutrophils and monocytes. Blood 112, 935-945. doi: 10.1182/blood-2007-12-077917

\section{ETHICS STATEMENT}

The animal study was reviewed and approved by the Experimental Animal Ethics Committee of Pharmacy, Fudan University (2019-03-HSYY-ZJ-01).

\section{AUTHOR CONTRIBUTIONS}

XLi and XLiu designed and performed the experiments, collected, and analyzed the data. XLi wrote the first draft of the manuscript. $\mathrm{PH}$ analyzed the data and revised the manuscript. YH revised the manuscript. JZ designed the experiments and revised the manuscript. All authors read and approved the final version of the manuscript.

\section{FUNDING}

This research was supported by the Shanghai Municipal Science and Technology Commission (grant nos. 17DZ1910402 and 19411964900) and the Major Research and Development Project of Innovative Drugs, Ministry of Science and Technology of China (grant no. 2017ZX09304005).

\section{SUPPLEMENTARY MATERIAL}

The Supplementary Material for this article can be found online at: https://www.frontiersin.org/articles/10.3389/fgene. 2021.563516/full\#supplementary-material

Supplementary Table 1 | List of differentially expressed proteins identified between CON and INF sample group of mice lung.

Supplementary Table 2 | Details of the GO, KEGG, and REACTOME pathways obtained from gProfiler.

Dikshit, N., Kale, S. D., Khameneh, H. J., Balamuralidhar, V., Tang, C. Y., Kumar, P., et al. (2018). NLRP3 inflammasome pathway has a critical role in the host immunity against clinically relevant Acinetobacter baumannii pulmonary infection. Mucosal Immunol. 11, 257-272. doi: 10.1038/mi.2017.50

Feng, Z., Jia, X., Adams, M. D., Ghosh, S. K., Bonomo, R. A., and Weinberg, A. (2014). Epithelial innate immune response to Acinetobacter baumannii challenge. Infect. Immun. 82, 4458-4465. doi: 10.1128/IAI.01897-14

Ferrer-Navarro, M., Strehlitz, A., Medina, E., and Vila, J. (2018). Changed expression of cytoskeleton proteins during lung injury in a mouse model of Streptococcus pneumoniae infection. Front. Microbiol. 9:928. doi: 10.3389/fmicb. 2018.00928

Garcia-Patino, M. G., Garcia-Contreras, R., and Licona-Limon, P. (2017). The immune response against Acinetobacter baumannii, an emerging pathogen in nosocomial infections. Front. Immunol. 8:441. doi: 10.3389/fimmu.2017.00441

Haziot, A., He, Z., Riva, M., Björk, P., Swärd, K., Mörgelin, M., et al. (2016). CD14 is a co-receptor for TLR4 in the S100A9-induced pro-inflammatory response in monocytes. PLoS One 11:e0156377. doi: 10.1371/journal.pone.0156377

Herdendorf, T. J., Stapels, D. A. C., Rooijakkers, S. H. M., and Geisbrecht, B. V. (2020). Local structural plasticity of the Staphylococcus aureus evasion protein EapH1 enables engagement with multiple neutrophil serine proteases. J. Biol. Chem. 295, 7753-7762. doi: 10.1074/jbc.RA120.013601

Hood, M. I., Mortensen, B. L., Moore, J. L., Zhang, Y., Kehl-Fie, T. E., Sugitani, N., et al. (2012). Identification of an Acinetobacter baumannii zinc acquisition 
system that facilitates resistance to calprotectin-mediated zinc sequestration. PLoS Pathog 8:e1003068. doi: 10.1371/journal.ppat.1003068

Hordijk, P.L. (2006). Regulation of NADPH Oxidases. Circ. Res. 98, 453-462. doi: 10.1161/01.RES.0000204727.46710.5e

Hu, F. P., Guo, Y., Yang, Y., Zheng, Y. G., Wu, S., Jiang, X. F., et al. (2019). Resistance reported from China antimicrobial surveillance network (CHINET) in 2018. Eur. J. Clin. Microbiol. Infect. Dis. 38, 2275-2281. doi: 10.1007/s10096019-03673-1

Hughes, B. J., Hollers, J. C., Crockett-Torabi, E., and Smith, C. W. (1992). Recruitment of $\mathrm{CD} 11 \mathrm{~b} / \mathrm{CD} 18$ to the neutrophil surface and adherencedependent cell locomotion. J. Clin. Invest. 90, 1687-1696. doi: 10.1172/ jci116041

Kamoshida, G., Akaji, T., Takemoto, N., Suzuki, Y., Sato, Y., Kai, D., et al. (2020). Lipopolysaccharide-deficient Acinetobacter baumannii due to colistin resistance is killed by neutrophil-produced lysozyme. Front. Microbiol. 11:573. doi: $10.3389 /$ fmicb.2020.00573

Kamoshida, G., Kikuchi-Ueda, T., Nishida, S., Tansho-Nagakawa, S., Ubagai, T., and Ono, Y. (2018). Pathogenic bacterium Acinetobacter baumannii inhibits the formation of neutrophil extracellular traps by suppressing neutrophil adhesion. Front. Immunol. 9:178. doi: 10.3389/fimmu.2018.00178

Kamoshida, G., Kikuchi-Ueda, T., Tansho-Nagakawa, S., Nakano, R., Nakano, A., Kikuchi, H., et al. (2015). Acinetobacter baumannii escape from neutrophil extracellular traps (NETs). J. Infect. Chemother. 21, 43-49. doi: 10.1016/j.jiac. 2014.08.032

Karp, A. N., Huber, W., Sadowski, G. P., Charles, D. P., Hester, V. S., Lilley, S. K., et al. (2010). Addressing accuracy and precision issues in iTRAQ quantitation. Mol. Cell. Proteom 9, 1885-1897. doi: 10.1074/mcp.M900628-MCP200

Kawasaki, T., and Kawai, T. (2014). Toll-like receptor signaling pathways. Front. Immunol. 5:461.

Kim, C. H., Jeong, Y. J., Lee, J., Jeon, S. J., Park, S. R., Kang, M. J., et al. (2013). Essential role of toll-like receptor 4 in Acinetobacter baumannii-induced immune responses in immune cells. Microb. Pathog 54, 20-25. doi: 10.1016/j. micpath.2012.08.008

Knapp, S., Wieland, C. W., Florquin, S., Pantophlet, R., Dijkshoorn, L., Tshimbalanga, N., et al. (2006). Differential roles of CD14 and toll-like receptors 4 and 2 in murine Acinetobacter pneumonia. Am. J. Respir. Crit. Care Med. 173, 122-129. doi: 10.1164/rccm.200505-730OC

Kosciuczuk, E. M., Lisowski, P., Jarczak, J., Strzalkowska, N., Jozwik, A., Horbanczuk, J., et al. (2012). Cathelicidins: family of antimicrobial peptides. Mol. Biol. Rep. 39, 10957-10970. doi: 10.1007/s11033-012-1997-x

Langer, H. F., and Chavakis, T. (2009). Leukocyte-endothelial interactions in inflammation. J. Cell Mol. Med. 13, 1211-1220. doi: 10.1111/j.1582-4934.2009. 00811.x

Li, J., Nation, R. L., Turnidge, J. D., Milne, R. W., Coulthard, K., Rayner, C. R., et al. (2006). Colistin: the re-emerging antibiotic for multidrug-resistant Gramnegative bacterial infections. Lancet Infect. Dis. 6, 589-601. doi: 10.1016/s14733099(06)70580-1

Liu, C., Shan, B., Qi, J., and Ma, Y. (2017). Systemic Responses of MultidrugResistant Pseudomonas aeruginosa and Acinetobacter baumannii following exposure to the antimicrobial peptide cathelicidin-BF imply multiple intracellular targets. Front. Cell Infect. Microbiol. 7:466. doi: 10.3389/fcimb. 2017.00466

Mahdi, L., Mahdi, N., Al-Kakei, S., Musafer, H., Al-Joofy, I., Essa, R., et al. (2018). Treatment strategy by lactoperoxidase and lactoferrin combination: Immunomodulatory and antibacterial activity against multidrug-resistant Acinetobacter baumannii. Microb. Pathog 114, 147-152. doi: 10.1016/j.micpath. 2017.10.056

Moore, J. L., Becker, K. W., Nicklay, J. J., Boyd, K. L., Skaar, E. P., and Caprioli, R. M. (2014). Imaging mass spectrometry for assessing temporal proteomics: analysis of calprotectin in Acinetobacter baumannii pulmonary infection. Proteomics 14, 820-828. doi: 10.1002/pmic.201300046

Muller, W. A. (2011). Mechanisms of leukocyte transendothelial migration. Annu. Rev. Pathol. 6:323. doi: 10.1146/annurev-pathol-011110-130224

Noto, M. J., Boyd, K. L., Burns, W. J., Varga, M. G., Peek, R. M., and Skaar, E. P. (2015). Toll-like receptor 9 contributes to defense against Acinetobacter baumannii infection. Infect. Immun. 83, 4134-4141. doi: 10.1128/iai.004 10-15

Nourshargh, S., Hordijk, P. L., and Sixt, M. (2010). Breaching multiple barriers: leukocyte motility through venular walls and the interstitium. Nat. Rev. Mol. Cell Biol. 11, 366-378. doi: 10.1038/nrm2889

Park, B. S., and Lee, J. O. (2013). Recognition of lipopolysaccharide pattern by TLR4 complexes. Exp. Mol. Med. 45:e66. doi: 10.1038/emm.2013.97

Qiu, H., Kuolee, R., Harris, G., and Chen, W. (2009). Role of NADPH phagocyte oxidase in host defense against acute respiratory Acinetobacter baumannii infection in mice. Infect. Immun. 77, 1015-1021. doi: 10.1128/IAI.01029-08

Reinhardt, A., Wehle, M., Geissner, A., Crouch, E. C., Kang, Y., Yang, Y., et al. (2016). Structure binding relationship of human surfactant protein D and various lipopolysaccharide inner core structures. J. Struct. Biol. 195, 387-395. doi: $10.1016 /$ j.jsb.2016.06.019

Roberts, A. W., Kim, C., Zhen, L., Lowe, J. B., Kapur, R., Petryniak, B., et al. (1999). Deficiency of the hematopoietic cell-specific rho family GTPase Rac2 Is characterized by abnormalities in neutrophil function and host defense. Immunity 10, 183-196. doi: 10.1016/s1074-7613(00)80019-9

Sato, Y., Unno, Y., Miyazaki, C., Ubagai, T., and Ono, Y. (2019). Multidrugresistant Acinetobacter baumannii resists reactive oxygen species and survives in macrophages. Sci. Rep. 9:17462. doi: 10.1038/s41598-019-53846-3

Schimmel, L., Heemskerk, N., and Van Buul, J. D. (2017). Leukocyte transendothelial migration: a local affair. Small GTPases 8, 1-15. doi: 10.1080/ 21541248.2016.1197872

Seddigh, P., Bracht, T., Molinier-Frenkel, V., Castellano, F., Kniemeyer, O., Schuster, M., et al. (2017). Quantitative analysis of proteome modulations in alveolar epithelial type II cells in response to Pulmonary Aspergillus fumigatus infection. Mol. Cell Proteomics 16, 2184-2198. doi: 10.1074/mcp.RA117.000072

Spencer, J. J., Pitts, R. E., Pearson, R. A., and King, L. B. (2018). The effects of antimicrobial peptides WAM-1 and LL-37 on multidrug-resistant Acinetobacter baumannii. Pathog Dis 76, fty007. doi: 10.1093/femspd/fty007

Steinwede, K., Maus, R., Bohling, J., Voedisch, S., Braun, A., Ochs, M., et al. (2012). Cathepsin G and neutrophil elastase contribute to lung-protective immunity against mycobacterial infections in mice. J. Immunol. 188, 4476-4487. doi: 10.4049/jimmunol.1103346

van Faassen, H., Kuolee, R., Harris, G., Zhao, X., Conlan, J. W., and Chen, W. (2007). Neutrophils play an important role in host resistance to respiratory infection with Acinetobacter baumannii in mice. Infect. Immun. 75, 5597-5608. doi: 10.1128/IAI.00762-07

Varnum, S. M., Webb-Robertson, B. J., Pounds, J. G., Moore, R. J., Smith, R. D., Frevert, C. W., et al. (2012). Proteomic analysis of bronchoalveolar lavage fluid proteins from mice infected with Francisella tularensis ssp. novicida. J. Proteome Res. 11, 3690-3703. doi: 10.1021/pr3001767

Vermeer, P. D., Denker, J., Estin, M., Moninger, T. O., Keshavjee, S., Karp, P., et al. (2009). MMP9 modulates tight junction integrity and cell viability in human airway epithelia. Am. J. Physiol. Lung Cell Mol. Physiol. 296, L751-L762. doi: 10.1152/ajplung.90578.2008

Vestweber, D. (2010). Adhesion and signaling molecules controlling the transmigration of leukocytes through endothelium. Immunol. Rev. 218, 178196. doi: 10.1111/j.1600-065x.2007.00533.x

Zhou, H., Liao, J., Aloor, J., Nie, H., Wilson, B. C., Fessler, M. B., et al. (2013). $\mathrm{CD} 11 \mathrm{~b} / \mathrm{CD} 18$ (Mac-1) is a novel surface receptor for extracellular doublestranded RNA to mediate cellular inflammatory responses. J. Immunol. 190, 115-125. doi: 10.4049/jimmunol.1202136

Conflict of Interest: The authors declare that the research was conducted in the absence of any commercial or financial relationships that could be construed as a potential conflict of interest.

Copyright $(2021 \mathrm{Li}$, Liu, Horvatovich, Hu and Zhang. This is an open-access article distributed under the terms of the Creative Commons Attribution License (CC BY). The use, distribution or reproduction in other forums is permitted, provided the original author(s) and the copyright owner(s) are credited and that the original publication in this journal is cited, in accordance with accepted academic practice. No use, distribution or reproduction is permitted which does not comply with these terms. 